\title{
SCATTERED HOMOCLINICS TO A CLASS OF TIME-RECURRENT HAMILTONIAN SYSTEMS
}

\author{
Gregory S. SPradLin ${ }^{1}$
}

\begin{abstract}
A second-order Hamiltonian system with time recurrence is studied. The recurrence condition is weaker than almost periodicity. The existence is proven of an infinite family of solutions homoclinic to zero whose support is spread out over the real line.
\end{abstract}

Mathematics Subject Classification. 34C37, 47J30.

Received December 5, 2005. Revised May 25, 2006.

Published online June 5, 2007.

\section{INTRODUCTION}

In this paper we study a second-order Hamiltonian system with a time recurrence property that is weaker than almost periodicity. The zero function is a hyperbolic fixed point solution. We prove the existence of infinitely many solutions homoclinic to zero, that is, solutions $v$ with $v(t) \rightarrow 0$ and $v^{\prime}(t) \rightarrow 0$ as $t \rightarrow \pm \infty$. The solutions are scattered infinitely far to the left and to the right along the real line.

The Hamiltonian system has the form

$$
-u^{\prime \prime}+u=W^{\prime}(t, u)
$$

where $u: \mathbb{R} \rightarrow \mathbb{R}^{N}, W \in C^{1}\left(\mathbb{R} \times \mathbb{R}^{N}, \mathbb{R}\right), W^{\prime}(t, u) \equiv \nabla_{q} W(t, q) \equiv<\frac{\partial W}{\partial q_{1}}, \ldots, \frac{\partial W}{\partial q_{N}}>$, and $W(t, u)$ is a "superquadratic" function of $u$, and satisfies a time recurrence property in $t$ that is weaker than almost periodicity. To be precise, let $N \in \mathbb{N}^{+}$and let $W$ satisfy

$\left(W_{1}\right) W \in C^{1}\left(\mathbb{R} \times \mathbb{R}^{N}, \mathbb{R}\right)$ and $W^{\prime}(t, \cdot)$ is locally Lipschitz, uniformly in $t$.

$\left(W_{2}\right) W(t, 0)=0$ for all $t \in \mathbb{R}$. $\left|W^{\prime}(t, q)\right| /|q| \rightarrow 0$ as $|q| \rightarrow 0$, uniformly in $t$.

$\left(W_{3}\right)$ There exists $\mu>2$ with $W^{\prime}(t, q) \cdot q \geq \mu W(t, q) \geq 0$ for all $(t, q) \in \mathbb{R} \times \mathbb{R}^{N}$. There exists $(\bar{t}, \bar{q}) \in$ $\mathbb{R} \times\left(\mathbb{R}^{N} \backslash\{0\}\right)$ such that $\frac{1}{2}|\bar{q}|^{2}-W(\bar{t}, \bar{q}) \leq 0$.

$\left(W_{4}\right)$ There exists a sequence $\left(t_{m}\right) \subset \mathbb{R}^{+}$such that $W^{\prime}\left(t-t_{m}, q\right) \rightarrow W^{\prime}(t, q)$ as $m \rightarrow \infty$ for all $t \in \mathbb{R}, q \in \mathbb{R}^{N}$. These assumptions are the same as in [2]. The reader is referred to that paper for more background on the equation. Both [2] and this paper prove that (1.1) has infinitely many solutions homoclinic to zero. Both papers prove a stronger result than [5]. In [5], $W$ is assumed to be in "factored" form $\alpha(t) G(q)$, with $\alpha$ almost periodic in $t$, which is a stronger assumption than $\left(W_{4}\right)$. Also, [5] proves the existence of only one nontrivial solution homoclinic to zero.

Keywords and phrases. Mountain pass theorem, Palais-Smale sequences, almost periodic, Hamiltonian system.

1 Department of Mathematics, Embry-Riddle University, Daytona Beach, Florida 32114-3900, USA; spradlig@erau.edu

(C) EDP Sciences, SMAI 2007 
In [2], it is proven that the set of solutions of (1.1) homoclinic to zero is uncountable, or there exists an infinite familty of "multibump" solutions, which resemble the sums of translates of a particular homoclinic solution. This is done by assuming the set of solutions homoclinic to zero to be countable, then finding one critical point of the corresponding functional with local "mountain-pass" geometry, then "gluing" that critical point to translates of itself. This paper also proves that (1.1) has infinitely many solutions homoclinic to zero, relying on the recurrence property of $W$ rather than a multibump construction. We obtain the following result, which is independent of the very strong result of [2].

Theorem 1.1. If $W$ satisfies $\left(W_{1}\right)-\left(W_{4}\right)$, then there exist $r_{0}>0$, sequences $\left(t_{m}^{+}\right)$and $\left(t_{m}^{-}\right)$with $t_{m}^{+} \rightarrow \infty$ and $t_{m}^{-} \rightarrow-\infty$ as $m \rightarrow \infty$, and sequences of solutions $\left(v_{m}^{+}\right),\left(v_{m}^{-}\right)$of $(1.1)$ homoclinic to zero with $\left|v_{m}^{+}\left(t_{m}^{+}\right)\right|>r_{0}$ and $\left|v_{m}^{-}\left(t_{m}^{-}\right)\right|>r_{0}$ for all $m$.

The proof of the theorem is shorter and simpler than that of [2], but does not describe the structure of solutions in such detail.

This paper is organized as follows: In Section 2 the variational setting of the problem is set up, the theorem is proved for a special case, and some technical lemmas are proven. Section 3 completes the proof for the more difficult case.

\section{VARIATIONAL SETTING AND PRELIMINARY LEMMAS}

Define $E=W^{1,2}\left(\mathbb{R}, \mathbb{R}^{N}\right)$. Let $(\cdot, \cdot)$ be the standard inner product on $E$, that is, $(u, w)=\int_{\mathbb{R}} u^{\prime} \cdot w^{\prime}+u \cdot w \mathrm{~d} t$, with corresponding norm $\|u\|^{2}=(u, u)$. Define the functional $I: E \rightarrow \mathbb{R}$ by $I(u)=\frac{1}{2}\|u\|^{2}-\int_{\mathbb{R}} W(t, u) \mathrm{d} t$. By $\left(W_{1}\right)-\left(W_{2}\right), I$ is Fréchet differentiable, with $I^{\prime}(u) w=(u, w)-\int_{\mathbb{R}} W^{\prime}(t, u) \cdot w \mathrm{~d} t$, and $I^{\prime}$ is locally Lipschitz. Critical points of $I$ correspond exactly to solutions of (1.1) homoclinic to zero. By $\left(W_{2}\right), I(u)=\frac{1}{2}\|u\|^{2}-o\left(\|u\|^{2}\right)$ as $\|u\| \rightarrow 0$. By $\left(W_{3}\right)$, there exists $u_{0} \in E$ with $I\left(u_{0}\right)<0$. Therefore, $I$ satisfies the geometric conditions of the Mountain Pass Theorem of Ambrosetti and Rabinowitz [3]. That is, the set of "mountain-pass curves"

$$
\Gamma=\{\gamma \in C([0,1], E) \mid \gamma(0)=0, I(\gamma(1))<0\}
$$

is nonempty, and the mountain-pass value

$$
c=\inf _{\gamma \in \Gamma[0,1]} \max _{[0, \gamma(\theta))}
$$

is positive. $I$ does not satisfy the Palais-Smale condition, however, so the Mountain Pass Theorem cannot be applied. The Palais-Smale condition holds if any sequence $\left(u_{m}\right) \subset E$ with $I\left(u_{m}\right)$ convergent and $I^{\prime}\left(u_{m}\right) \rightarrow 0$ has a convergent subsequence. $I$ does not satisfy the Palais-Smale condition, because the domain $\mathbb{R}$ is unbounded. Even if, for example, $W$ is periodic in $t$, the Palais-Smale condition fails even modulo the periodicity: suppose $W$ is 1-periodic in $t$, and $v$ is a nontrivial solution of (1.1) homoclinic to zero. Define the translation operator $\tau$ as follows: for a function $u$ defined on $\mathbb{R}$ and real $a$, let $\tau_{a} u$ be $u$ shifted $a$ units to the right; that is, $\left(\tau_{a} u\right)(t)=$ $u(t-a)$. Then the sequence $\left(v+\tau_{n} v\right)$ satisfies $I\left(v+\tau_{n} v\right) \rightarrow 2 I(v)$ and $I^{\prime}\left(v+\tau_{n} v\right) \rightarrow 0$ as $n \rightarrow \infty$, but the sequence has no convergent subsequence, even if we are allowed to shift functions by integer multiples of a period.

With $\mu$ as in $\left(W_{3}\right)$, define

$$
B=1+\frac{200 c \mu}{\mu-2}
$$

Let $r_{0}>0$ be small enough that

$$
\left|W^{\prime}(t, q)\right| \leq \frac{1}{64}|q|
$$

for all $(t, q) \in \mathbb{R} \times \mathbb{R}^{N}$ with $|q| \leq r_{0}$. This is possible by $\left(W_{2}\right)$. Finally, let $M$ be an integer with

$$
M>\frac{4 B^{2}}{r_{0}^{2}} \text {. }
$$


Define $\mathcal{T}: \mathbb{R} \rightarrow \mathbb{R}^{+}$by

$$
\mathcal{T}(t)=\inf \left\{\left\|I^{\prime}(u)\right\| \mid\|u\| \leq B,\|u\|_{L^{\infty}(t-M, t+M)} \geq r_{0}\right\}
$$

Then one of the following alternatives holds:

$$
\begin{aligned}
& \text { Case I: } \mathcal{T}(t)=0 \text { for all } t \in \mathbb{R} \text {, or } \\
& \text { Case II: } \mathcal{T}(\tilde{t})>0 \text { for some } \tilde{t} \in \mathbb{R} .
\end{aligned}
$$

Suppose Case I holds. The precise values of $r_{0}, B$, and $M$ are unimportant in this case, only that they are positive. Let $t_{0} \in \mathbb{R}$ be arbitrary. There exists a sequence $\left(u_{m}\right)$ with $\left\|I^{\prime}\left(u_{m}\right)\right\| \rightarrow 0$ as $m \rightarrow \infty,\left\|u_{m}\right\| \leq B$ for all $m$, and $\|u\|_{L^{\infty}\left(t_{0}-M, t_{0}+M\right)} \geq r_{0}$ for all $m$. Along a subsequence (also denoted $\left.\left(u_{m}\right)\right),\left(u_{m}\right)$ converges weakly and in $L_{\text {loc }}^{\infty}\left(\mathbb{R}, \mathbb{R}^{N}\right)$ to $\bar{u}$ with $\|\bar{u}\| \leq B$ and $\|\bar{u}\|_{L^{\infty}\left(t_{0}-M, t_{0}+M\right)} \geq r_{0}$. By arguments of [1], or arguments from Lemma 2.1 of this paper, $\bar{u}$ is a critical point of $I$, hence a solution of (1.1). $t_{0}$ was arbitrary, so Theorem 1.1 holds.

The rest of this paper is devoted to Case II. First, if $\mathcal{T}$ is positive somewhere, it is bounded away from zero on a sequence of $t$ 's that approaches infinity:

Lemma 2.1. If $\mathcal{T}(\tilde{t})>0$, then there exist $\delta>0$ and $K \in \mathbb{N}$ with $m \geq K \Rightarrow \mathcal{T}\left(\tilde{t}+t_{m}\right)>\delta$.

Proof. Suppose otherwise, that there exist $\tilde{t} \in \mathbb{R}$ with $\mathcal{T}(\tilde{t})>0$ and a subsequence of $\left(t_{m}\right)$ (also denoted $\left(t_{m}\right)$ ) with $\mathcal{T}\left(\tilde{t}+t_{m}\right) \rightarrow 0$. Pick a sequence $\left(u_{m}\right) \subset E$ with $\left\|u_{m}\right\| \leq B$ and

$$
\left\|u_{m}\right\|_{L^{\infty}\left(\tilde{t}+t_{m}-M, \tilde{t}+t_{m}+M\right)} \geq r_{0}
$$

for all $m$, and $\left\|I^{\prime}\left(u_{m}\right)\right\| \rightarrow 0$. $\left(\tau_{-t_{m}} u_{m}\right)$ converges weakly and in $L_{l o c}^{\infty}\left(\mathbb{R}, \mathbb{R}^{N}\right)$ along a subsequence (also denoted $\left.\left(\tau_{-t_{m}} u_{m}\right)\right)$ to $\bar{u} \in E$ with $\|\bar{u}\| \leq B$ and

$$
\|\bar{u}\|_{L^{\infty}(\tilde{t}-M, \tilde{t}+M)} \geq r_{0}
$$

We will show that $I^{\prime}(\bar{u})=0$, contradicting the fact that $\mathcal{T}(\tilde{t})>0$. Let $w \in E$ be arbitrary with $\|w\|=1$. Then $I^{\prime}\left(u_{m}\right)\left(\tau_{t_{m}} w\right) \rightarrow 0$. Now

$$
\begin{aligned}
I^{\prime}\left(u_{m}\right)\left(\tau_{t_{m}} w\right)-I^{\prime}(\bar{u}) w & =\left(u_{m}, \tau_{t_{m}} w\right)-(\bar{u}, w)+\int_{\mathbb{R}} W^{\prime}(t, \bar{u}) w(t) \mathrm{d} t-\int_{\mathbb{R}} W^{\prime}\left(t, u_{m}\right) \tau_{t_{m}} w(t) \mathrm{d} t \\
& =\left(\tau_{-t_{m}} u_{m}, w\right)-(\bar{u}, w)+\int_{\mathbb{R}} W^{\prime}(t, \bar{u}) w(t)-W^{\prime}\left(t+t_{m}, u_{m}\left(t+t_{m}\right)\right) w(t) \mathrm{d} t \\
& =\left(\tau_{-t_{m}} u_{m}-\bar{u}, w\right)+\int_{\mathbb{R}}\left(W^{\prime}(t, \bar{u})-W^{\prime}\left(t+t_{m}, u_{m}\left(t+t_{m}\right)\right)\right) w(t) \mathrm{d} t
\end{aligned}
$$

$\tau_{-t_{m}} u_{m} \rightarrow \bar{u}$ weakly, so the inner product at the end of (2.10) goes to 0 as $m \rightarrow \infty$. Now let $\epsilon>0$. Let $R>0$ with

$$
\int_{|t|>R}|w|^{2} \mathrm{~d} t<\epsilon^{2}
$$

Since $\tau_{-t_{m}} u_{m} \rightarrow \bar{u}$ in $L^{\infty}\left(\mathbb{R}, \mathbb{R}^{N}\right),\left(W_{1}\right)$ and $\left(W_{4}\right)$ imply that

$$
\left(W^{\prime}(t, \bar{u}(t))-W^{\prime}\left(t+t_{m}, u_{m}\left(t+t_{m}\right)\right)\right) w(t) \rightarrow 0
$$

as $m \rightarrow \infty$ for all $t \in \mathbb{R}$. Since $\left(u_{m}\right)$ is bounded in $L^{\infty}\left(\mathbb{R}, \mathbb{R}^{N}\right)$ and $w$ is continuous, there exists $C_{2}>0$ with

$$
\left|\left(W^{\prime}(t, \bar{u}(t))-W^{\prime}\left(t+t_{m}, u_{m}\left(t+t_{m}\right)\right)\right) w(t)\right| \leq C_{2}
$$

for all $t \in \mathbb{R}, m \in \mathbb{N}$. By the Dominated Convergence Theorem,

$$
\int_{-R}^{R}\left(W^{\prime}(t, \bar{u}(t))-W^{\prime}\left(t+t_{m}, u_{m}\left(t+t_{m}\right)\right)\right) w(t) \rightarrow 0
$$


as $m \rightarrow \infty$. By $\left(W_{2}\right)$, since $\left(u_{m}\right)$ is bounded in $L^{\infty}\left(\mathbb{R}, \mathbb{R}^{N}\right)$, there exists $C>0$ with

$$
\left|W^{\prime}(t, \bar{u}(t))\right| \leq C|\bar{u}(t)| \text { and }\left|W^{\prime}\left(t, u_{m}(t)\right)\right| \leq C\left|u_{m}(t)\right|
$$

for all $t \in \mathbb{R}$ and $m \in \mathbb{N}$. By the Cauchy-Schwarz Inequality, for all $m$,

$$
\begin{aligned}
\int_{|t| \geq R} & \left(W^{\prime}(t, \bar{u}(t))-W^{\prime}\left(t+t_{m}, u_{m}\left(t+t_{m}\right)\right)\right) w(t) \mathrm{d} t \\
& \leq\left(\int_{|t| \geq R}\left|W^{\prime}(t, \bar{u}(t))-W^{\prime}\left(t+t_{m}, u_{m}\left(t+t_{m}\right)\right)\right|^{2} \mathrm{~d} t\right)^{\frac{1}{2}}\left(\int_{|t| \geq R}|w|^{2} \mathrm{~d} t\right)^{\frac{1}{2}} \\
& \leq \epsilon\left(\int_{|t| \geq R}\left|C \bar{u}(t)+C u_{m}\left(t+t_{m}\right)\right|^{2} \mathrm{~d} t\right)^{\frac{1}{2}} \\
& \leq 2 C \epsilon\left(\int_{\mathbb{R}}|\bar{u}(t)|^{2}+\left|u_{m}\left(t+t_{m}\right)\right|^{2}\right)^{\frac{1}{2}} \\
& \leq 4 B C \epsilon
\end{aligned}
$$

Thus, by (2.14) and (2.16),

$\epsilon>0$ was arbitrary, so

$$
\lim \sup _{m \rightarrow \infty}\left|I^{\prime}\left(u_{m}\right)\left(\tau_{t_{m}} w\right)-I^{\prime}(\bar{u}) w\right| \leq 4 B C \epsilon
$$

$$
\lim _{m \rightarrow \infty}\left|I^{\prime}\left(u_{m}\right)\left(\tau_{t_{m}} w\right)-I^{\prime}(\bar{u}) w\right|=0
$$

Since $I^{\prime}\left(u_{m}\right)\left(\tau_{t_{m}} w\right) \rightarrow 0, I^{\prime}(\bar{u}) w=0 . w \in E$ was arbitrary with $\|w\|=1$, so $I^{\prime}(\bar{u})=0$. This contradicts the assumption that $\mathcal{T}(\tilde{t})>0$. Lemma 2.1 is proven.

For $u \in E$, define $\nabla I(u)$ to be the gradient of $u$, satisfying $(\nabla I(u), w)=I^{\prime}(u) w$ for all $w \in E$. Let $\varphi: E \rightarrow[0,1]$ be locally Lipschitz continuous with $\varphi(u)=1$ if $I(u) \geq-1$ and $\varphi(u)=0$ if $I(u) \leq-2$. Define the gradient vector flow $\eta$ to be the solution of the initial value problem

$$
\frac{\mathrm{d} \eta}{\mathrm{d} s}=-\varphi(u) \nabla I(u), \eta(0, u)=u
$$

$\eta$ is well-defined on $\mathbb{R}^{+} \times E$ (see [4]). We will need several lemmas about $\eta$. First, for the functions $u$ that we will be most interested in, there is an a priori bound on $\eta(s, u)$ for $s \geq 0$ :

Lemma 2.2. If $u \in E$ with $I(u) \leq 2 c$ and $\lim _{s \rightarrow \infty} I(\eta(s, u))>0$, then $\|u\| \leq B$, where $B$ is from (2.3).

Proof. For all $w \in E$ with $I(w) \leq 2 c$ and $\|w\| \geq B / 2$,

$$
\begin{aligned}
\left\|I^{\prime}(w)\right\| & \geq-\frac{I^{\prime}(w) w}{\|w\|}=\frac{-\|w\|^{2}+\int_{\mathbb{R}} W^{\prime}(t, w) w \mathrm{~d} t}{\|w\|} \\
& \geq \frac{-\|w\|^{2}+\mu \int_{\mathbb{R}} W(t, w) \mathrm{d} t}{\|w\|}=\frac{-\|w\|^{2}+\frac{\mu}{2}\|w\|^{2}-\mu I(w)}{\|w\|} \\
& \geq\left(\frac{\mu}{2}-1\right)\|w\|-\frac{2 c \mu}{\|w\|} \geq \frac{1}{4}\left(\frac{\mu}{2}-1\right) B
\end{aligned}
$$

by $\left(W_{3}\right)$ and the definition of $B((2.3))$. Now let $u \in E$ with $I(u) \leq 2 c, \lim _{s \rightarrow \infty} I(s, u)>0$, and $\|u\| \geq B$. We will arrive at a contradiction. Define $\eta(s) \equiv \eta(s, u)$. If $\|\eta(s)\| \geq B / 2$ for all $s \geq 0$, then by (2.20), $\frac{\mathrm{d}}{\mathrm{d} s} I(\eta(s))=-\left\|I^{\prime}(\eta(s))\right\|^{2} \leq-\frac{1}{16}\left(\frac{\mu}{2}-1\right)^{2} B^{2}$ for all $s>0$, so $I(\eta(s)) \rightarrow-\infty$ as $s \rightarrow \infty$, contradicting our 
assumption on $u$. Therefore there exists $s>0$ with $\|\eta(s)\| \leq B / 2$, and we may pick $0<s_{1}<s_{2}$ with $\left\|\eta\left(s_{1}\right)\right\|=B_{1},\left\|\eta\left(s_{2}\right)\right\|=B_{1} / 2$, and $\|\eta(s)\| \in(B / 2, B)$ for all $s \in\left(s_{1}, s_{2}\right)$. Then

$$
\begin{aligned}
2 c & \geq I\left(\eta\left(s_{1}\right)\right)-I\left(\eta\left(s_{2}\right)\right)=-\int_{s_{1}}^{s_{2}} \frac{\mathrm{d}}{\mathrm{d} s} I(\eta) \mathrm{d} s \\
& =\int_{s_{1}}^{s_{2}}\left\|I^{\prime}(\eta)\right\|^{2} \mathrm{~d} s \geq \frac{1}{16}\left(s_{2}-s_{1}\right)\left(\frac{\mu}{2}-1\right)^{2} B^{2} .
\end{aligned}
$$

On the other hand, the Cauchy-Schwarz Inequality yields

$$
\begin{aligned}
B / 2 & \leq\left\|\eta\left(s_{1}\right)-\eta\left(s_{2}\right)\right\|=\left\|\int_{s_{1}}^{s_{2}} \frac{\mathrm{d} \eta}{\mathrm{d} s} \mathrm{~d} s\right\| \leq \int_{s_{1}}^{s_{2}}\left\|\frac{\mathrm{d} \eta}{\mathrm{d} s}\right\| \mathrm{d} s \\
& \leq \sqrt{s_{2}-s_{1}}\left(\int_{s_{1}}^{s_{2}}\left\|\frac{\mathrm{d} \eta}{\mathrm{d} s}\right\|^{2} \mathrm{~d} s\right)^{\frac{1}{2}} \leq \sqrt{2 c} \sqrt{s_{2}-s_{1}} .
\end{aligned}
$$

Therefore

$$
\frac{128 c}{B^{2}(\mu-2)} \geq s_{2}-s_{1} \geq \frac{B^{2}}{8 c}, \quad B^{4}<\frac{1024 c^{2}}{(\mu-2)^{2}},
$$

contradicting the definition of $B((2.3))$. Lemma 2.2 is proven.

Lemma 2.2 is needed for the following:

Lemma 2.3. There exists $a>0$ such that if $I(u) \leq 2 c$ and $\lim _{s \rightarrow \infty} I(\eta(s, u))>0$, then

$$
\left\|I^{\prime}(\eta(s, u))\right\|^{3} \leq a\left(I(u)-\lim _{s \rightarrow \infty} I(\eta(s, u))\right)
$$

for all $s \geq 0$.

Proof. If $I^{\prime}(u)=0$, then both sides of the inequality (2.24) are zero, so assume $I^{\prime}(u) \neq 0$. Define $\eta \equiv \eta(s) \equiv$ $(s, u)$. By Lemma $2.2,\|\eta(s)\| \leq B$ for all $s \geq 0$. $I^{\prime}$ is Lipschitz on bounded subsets of $E$, so there exists $\beta>0$ with $\left\|I^{\prime}(w)-I^{\prime}(y)\right\| \leq \beta\|w-y\|$ for all $w, y \in E$ with $\|w\|,\|y\| \leq B$. Since $I^{\prime}(0)=0,\left\|I^{\prime}(w)\right\| \leq \beta B$ for all $w \in E$ with $\|w\| \leq B$. Suppose that for some $s^{*}>0$,

$$
\left\|I^{\prime}\left(\eta\left(s^{*}\right)\right)\right\|>A_{1} \equiv 3 \sqrt[3]{B \beta^{2}\left(I(u)-\lim _{s \rightarrow \infty} I(\eta(s, u))\right)} .
$$

This will lead to a contradiction. Let

$$
A_{2}=\frac{A_{1}}{2 \beta^{2} B}
$$

Then for all $s \in\left[s^{*}, s^{*}+A_{2}\right]$,

$$
\left\|\eta(s)-\eta\left(s^{*}\right)\right\|=\left\|\int_{s^{*}}^{s} \frac{\mathrm{d} \eta}{\mathrm{d} s} \mathrm{~d} s\right\| \leq \int_{s^{*}}^{s}\left\|\frac{\mathrm{d} \eta}{\mathrm{d} s}\right\| \mathrm{d} s=\int_{s^{*}}^{s}\left\|I^{\prime}(\eta)\right\| \mathrm{d} s \leq \beta B A_{2} .
$$

So for all $s \in\left[s^{*}, s^{*}+A_{2}\right]$,

$$
\begin{aligned}
\left\|I^{\prime}(\eta(s))\right\| & \geq\left\|I^{\prime}\left(\eta\left(s^{*}\right)\right)\right\|-\left\|I^{\prime}(\eta(s))-I^{\prime}\left(\eta\left(s^{*}\right)\right)\right\| \\
& \geq\left\|I^{\prime}\left(\eta\left(s^{*}\right)\right)\right\|-\beta\left\|\eta\left(s^{*}\right)-\eta(s)\right\| \\
& \geq\left\|I^{\prime}\left(\eta\left(s^{*}\right)\right)\right\|-\beta^{2} B A_{2} \geq \frac{1}{2} A_{1} .
\end{aligned}
$$


So

$$
\begin{aligned}
I\left(\eta\left(s^{*}+A_{2}\right)\right) & =I\left(\eta\left(s^{*}\right)\right)+\left(I\left(\eta\left(s^{*}+A_{2}\right)\right)-I\left(\eta\left(s^{*}\right)\right)\right. \\
& =I\left(\eta\left(s^{*}\right)\right)+\int_{s^{*}}^{s^{*}+A_{2}} \frac{\mathrm{d}}{\mathrm{d} s} I(\eta(s)) \mathrm{d} s \\
& =I\left(\eta\left(s^{*}\right)\right)-\int_{s^{*}}^{s^{*}+A_{2}}\left\|I^{\prime}(\eta(s))\right\|^{2} \mathrm{~d} s \\
& \leq I(u)-\int_{s^{*}}^{s^{*}+A_{2}}\left(A_{1} / 2\right)^{2} \mathrm{~d} s \\
& =I(u)-\frac{1}{4} A_{1}^{2} A_{2}=I(u)-\frac{A_{1}^{3}}{8 B \beta^{2}} \\
& \leq I(u)-2\left(I(u)-\lim _{s \rightarrow \infty} I(\eta(s))\right) \\
& <I(u)-\left(I(u)-\lim _{s \rightarrow \infty} I(\eta(s))\right)=\lim _{s \rightarrow \infty} I(\eta(s)) .
\end{aligned}
$$

This is impossible. The assumption is false, and the lemma is proven, with $a=27 B \beta^{2}$.

Several more lemmas are needed. First, two simple lemmas regarding cutoff functions:

Lemma 2.4. Let $u \in E$ and $\varphi \in W^{1, \infty}(\mathbb{R}, \mathbb{R})$ with $0 \leq \varphi(t) \leq 1$ for all $t \in \mathbb{R}$ and $\left|\varphi^{\prime}\right| \leq d$ almost everywhere. Then $\|\varphi u\| \leq(1+d)\|u\|$.

Proof.

$$
\begin{aligned}
\|\varphi u\|^{2} & =\int_{\mathbb{R}} \varphi^{2}|u|^{2}+\left|\varphi^{\prime} u+\varphi u^{\prime}\right|^{2} \mathrm{~d} t \\
& \leq \int_{\mathbb{R}}|u|^{2}+\left|\varphi^{\prime}\right|^{2}|u|^{2}+2\left|\varphi^{\prime}\right| \varphi|u|\left|u^{\prime}\right|+\varphi^{2}\left|u^{\prime}\right|^{2} \mathrm{~d} t \\
& \leq \int_{\mathbb{R}}|u|^{2}+d^{2}|u|^{2}+2 d|u|\left|u^{\prime}\right|+\left|u^{\prime}\right|^{2} \mathrm{~d} t \\
& \leq \int_{\mathbb{R}}|u|^{2}+d^{2}|u|^{2}+d|u|^{2}+d\left|u^{\prime}\right|^{2}+\left|u^{\prime}\right|^{2} \mathrm{~d} t \\
& \leq\left(1+d+d^{2}\right) \int_{\mathbb{R}}|u|^{2}+\left|u^{\prime}\right|^{2} \mathrm{~d} t \leq(1+d)^{2}\|u\|^{2} .
\end{aligned}
$$

Lemma 2.5. Let $u, w \in E$ and $\varphi \in W^{1, \infty}(\mathbb{R}, \mathbb{R})$ with $0 \leq \varphi(t) \leq 1$ for all $t \in \mathbb{R}$ and $\left|\varphi^{\prime}\right| \leq d$ almost everywhere, then $|(\varphi u, w)-(u, \varphi w)| \leq d\|u\|\|w\|$.

Proof.

$$
\begin{aligned}
|(\varphi u, w)-(u, \varphi w)| & =\left|\int_{\mathbb{R}}(\varphi u)^{\prime} \cdot w^{\prime}-u^{\prime} \cdot(\varphi w)^{\prime} \mathrm{d} t\right| \\
& =\left|\int_{\mathbb{R}}\left(\varphi^{\prime} u+\varphi u^{\prime}\right) \cdot w^{\prime}-u^{\prime} \cdot\left(\varphi^{\prime} w+\varphi w^{\prime}\right) \mathrm{d} t\right| \\
& =\left|\int_{\mathbb{R}} \varphi^{\prime}\left(u \cdot w^{\prime}-u^{\prime} \cdot w\right) \mathrm{d} t\right| \leq d \int_{\mathbb{R}}|u|\left|w^{\prime}\right|+\left|u^{\prime}\right||w| \mathrm{d} t \leq d\|u\|\|w\|
\end{aligned}
$$

by the Cauchy-Schwarz Inequality. 
The next lemma, on the properties of $\nabla I$, is needed for Lemma 2.7. Define $\mathcal{W}: E \rightarrow E$ by

$$
(\mathcal{W}(u), w)=\int_{\mathbb{R}} W^{\prime}(t, u) \cdot w(t) \mathrm{d} t
$$

for all $u, w \in E . \nabla I$ has the form $\nabla I(u)=u-\mathcal{W}(u)$. Now

Lemma 2.6. If $u \in E$ with $\|u\|_{L^{\infty}(\mathbb{R})} \leq r_{0}$, then $\|\mathcal{W}(u)\|_{L^{\infty}(\mathbb{R})} \leq r_{0} / 2$.

Proof. Let $u \in E$ with $\|u\|_{L^{\infty}(\mathbb{R})} \leq r_{0}$. Define $t_{\max } \in \mathbb{R}$ by

$$
\|\mathcal{W}(u)\|_{W^{1,2}\left(t_{\max }-4, t_{\max }+4\right)}=\max \left\{\|\mathcal{W}(u)\|_{W^{1,2}(t-4, t+4)} \mid t \in \mathbb{R}\right\}
$$

and define

$$
W_{\max }=\|\mathcal{W}(u)\|_{W^{1,2}\left(t_{\max }-4, t_{\max }+4\right)} .
$$

Define the piecewise linear cutoff function $\varphi$ by

$$
\varphi(t)= \begin{cases}0 ; & t \leq t_{\max }-8 \\ 2-\frac{1}{4}\left(t_{\max }-t\right) ; & t_{\max }-8 \leq t \leq t_{\max }-4 \\ 1 ; & t_{\max }-4 \leq t \leq t_{\max }+4 \\ 2-\frac{1}{4}\left(t-t_{\max }\right) ; & t_{\max }+4 \leq t \leq t_{\max }+8 \\ 0 ; & t \geq t_{\max }+8 .\end{cases}
$$

Now

$$
W_{\max } \leq\|\varphi \mathcal{W}(u)\|=\sup _{\|w\|=1}(\varphi \mathcal{W}(u), w)
$$

By Lemmas 2.4 and 2.5,

$$
W_{\max } \leq \sup _{\|w\|=1}(\mathcal{W}(u), \varphi w)+\frac{1}{4}\|\mathcal{W}(u)\|_{W^{1,2}\left(t_{\max }-8, t_{\max }+8\right)} .
$$

By the definition of $W_{\max }$,

$$
\|\mathcal{W}(u)\|_{W^{1,2}\left(t_{\max }-8, t_{\max }+8\right)}^{2}=\|\mathcal{W}(u)\|_{W^{1,2}\left(t_{\max }-8, t_{\max }\right)}^{2}+\|\mathcal{W}(u)\|_{W^{1,2}\left(t_{\max }, t_{\max }+8\right)}^{2} \leq 2 W_{\max }^{2} .
$$

So

$$
W_{\max } \leq \sup _{\|w\|=1} \int_{\mathbb{R}} W^{\prime}(t, u) \cdot \varphi w+\frac{\sqrt{2}}{4} W_{\max }
$$

and by (2.4),

So

$$
\begin{aligned}
\frac{1}{2} W_{\max } & \leq \sup _{\|w\|=1} \frac{1}{64} \int_{t_{\max }-8}^{t_{\max }+8}|\varphi \| w| \mathrm{d} t \leq \sup _{\|w\|=1} \frac{r_{0}}{64} \int_{t_{\max }-8}^{t_{\max }+8}|w| \mathrm{d} t \\
& \leq \sup _{\|w\|=1} \frac{r_{0}}{64}(\sqrt{16})\left(\int_{t_{\max }-8}^{t_{\max }+8}|w|^{2} \mathrm{~d} t\right)^{\frac{1}{2}} \leq \frac{1}{16} r_{0} .
\end{aligned}
$$

and for all $t \in \mathbb{R}$,

$$
W_{\max } \leq \frac{1}{8} r_{0}
$$

$$
\|\mathcal{W}(u)\|_{W^{1,2}(t-4, t+4)} \leq \frac{1}{8} r_{0} .
$$


By the embedding $W^{1,2}(0,8) \subset L^{\infty}(0,8)$, with embedding constant

$$
\|u\|_{L^{\infty}(0,8)} \leq \frac{1}{1-e^{-16}}\|u\|_{W^{1,2}(0,8)} \leq 2\|u\|_{W^{1,2}(0,8)}
$$

for all $u \in W^{1,2}(0,8)$ (equality achieved when $u(t)=\mathrm{e}^{-t} \mathbf{a}$, for any nonzero vector $\mathbf{a} \in \mathbb{R}^{N}$ ), $|\mathcal{W}(t)|<r_{0} / 4$ for all $t \in \mathbb{R}$.

The next lemma is essential to our variational argument. It enables us to conclude that trajectories of the gradient vector flow that are localized along the real line converge to nonzero critical points of $I$.

Lemma 2.7. If $u \in E$ with $\eta(s, u) \nrightarrow 0$ as $s \rightarrow \infty$, then $\|u\|_{L^{\infty}(\mathbb{R})} \geq r_{0}$.

Proof. We will prove the contrapositive. Let $u \in E$ with $\|u\|_{L^{\infty}(\mathbb{R})}<r_{0}$. Then for all $s>0,\|\eta(s, u)\|_{L^{\infty}(\mathbb{R})}<r_{0}$ : the proof is indirect - otherwise, let $s^{\prime}=\min \left\{s>0 \mid\|\eta(s, u)\|_{L^{\infty}(\mathbb{R})}=r_{0}\right\}$. There exists $t^{\prime} \in \mathbb{R}$ with $\left|\eta\left(s^{\prime}, u\right)\left(t^{\prime}\right)\right|=r_{0}$ and $\frac{\mathrm{d}}{\mathrm{d} s}\left|\eta\left(s^{\prime}, u\right)\left(t^{\prime}\right)\right|^{2} \geq 0$. But then, by (2.32)-(2.33),

$$
\begin{aligned}
0 & \leq \frac{\mathrm{d}}{\mathrm{d} t}\left|\eta\left(s^{\prime}, u\right)\left(t^{\prime}\right)\right|^{2}=-2 \eta\left(s^{\prime}, u\right)\left(t^{\prime}\right) \cdot \nabla I\left(\eta\left(s^{\prime}, u\right)\right)\left(t^{\prime}\right) \\
& =-2 \eta\left(s^{\prime}, u\right)\left(t^{\prime}\right) \cdot\left[\eta\left(s^{\prime}, u\right)\left(t^{\prime}\right)-\mathcal{W}\left(\eta\left(s^{\prime}, u\right)\left(t^{\prime}\right)\right]\right. \\
& \leq-2\left|\eta\left(s^{\prime}, u\right)\left(t^{\prime}\right)\right|^{2}+2\left|\eta\left(s^{\prime}, u\right)\left(t^{\prime}\right)\right| \mid \mathcal{W}\left(\eta\left(s^{\prime}, u\right)\left(t^{\prime}\right) \mid\right. \\
& \leq-2 r_{0}^{2}+2 r_{0}\left(\frac{r_{0}}{2}\right)<0
\end{aligned}
$$

This is a contradiction. So $\|\eta(s, u)\|_{L^{\infty}(\mathbb{R})}<r_{0}$ for all $s>0$. Now define $\phi(s)=\|\eta(s, u)\|^{2}$. For all $s>0$, Lemma 2.6 and (2.4) imply

$$
\begin{aligned}
\frac{\mathrm{d}}{\mathrm{d} s} \phi(s) & =-2(\eta(s, u), \nabla I(\eta(s, u)))=-2 I^{\prime}(\eta(s, u)) \eta(s, u) \\
& =-2\|\eta(s, u)\|^{2}+2 \int_{\mathbb{R}} W^{\prime}(t, \eta(s, u)) \eta(s, u) \\
& \leq-2\|\eta(s, u)\|^{2}+\frac{1}{32} \int_{\mathbb{R}}|\eta(s, u)|^{2} \mathrm{~d} t \leq-\|\eta(s, u)\|^{2}=-\phi(s)
\end{aligned}
$$

so $\phi(s) \leq \mathrm{e}^{-s}$ for all $s>0$, and $\phi(s) \rightarrow 0$ as $s \rightarrow \infty$.

\section{Proof of Theorem 1.1}

Theorem 1.1 follows from the following proposition:

Proposition 3.1. Let $r_{0}$ be as in (2.4), and $A>0$. There exist $t^{+}>A, t^{-}<-A$, and homoclinic solutions $v^{+}$and $v^{-}$to (1.1) with

$$
\left|v^{+}\left(t^{+}\right)\right|>r_{0} \text { and }\left|v^{-}\left(t^{-}\right)\right|>r_{0} .
$$

Proof. we will prove the existence of $v^{+}$. The proof for $v^{-}$is similar. Either Case I or Case II of (2.7) holds. The existence of $v^{+}$in Case I is proven in the argument following (2.7), so assume Case II holds. Let $\tilde{t}$ and $\delta$ be as in Case II and Lemma 2.1. Taking a subsequence of $\left(t_{m}\right)$ if necessary, assume that for all $m \in \mathbb{N}$,

$$
\left(\|u\| \leq B \text { and }\|u\|_{L^{\infty}\left(\tilde{t}+t_{m}-M, \tilde{t}+t_{m}+M\right)} \geq r_{0}\right) \Rightarrow\left\|I^{\prime}(u)\right\|>\delta .
$$

Let $m^{*}$ be large enough so that

$$
\tilde{t}+t_{m^{*}}-M>A .
$$


Let $a$ be as in Lemma 2.3. Define

$$
\epsilon=\min \left(\frac{\delta^{3}}{2 a}, \frac{c}{3}, \frac{r_{0}^{6}}{8000 B^{3} a}\right)
$$

Let $\gamma_{0} \in \Gamma$ with

$$
\max _{\theta \in[0,1]} I\left(\gamma_{0}(\theta)\right)<c+\epsilon / 2
$$

Let $\bar{m}$ be large enough so that

$$
\begin{gathered}
\tilde{t}+t_{\bar{m}}-M>\tilde{t}+t_{m^{*}}+M \\
I\left(\tau_{t_{\bar{m}}} \gamma_{0}\right)(1)<0 \\
I\left(\tau_{t_{\bar{m}}} \gamma_{0}(\theta)\right)<c+\epsilon \text { for all } \theta \in[0,1]
\end{gathered}
$$

and

$$
\left|\tau_{t_{\bar{m}}} \gamma_{0}(t)\right|<r_{0} \text { for all } t<\tilde{t}+t_{m^{*}}+M, \theta \in[0,1] .
$$

Let $m^{\prime}>\bar{m}$ be large enough so that

$$
\tilde{t}+t_{m^{\prime}}-M>\tilde{t}+t_{\bar{m}}+M,
$$

and

There exists $\bar{\theta} \in[0,1]$ with

$$
\left|\tau_{t_{m^{\prime}}} \gamma_{0}(\theta)(t)\right|<r_{0} \text { for all } t>\tilde{t}+t_{m^{\prime}}-M, \theta \in[0,1] .
$$

Define

$$
\lim _{s \rightarrow \infty} I\left(\eta\left(s, \tau_{t_{\bar{m}}} \gamma_{0}(\bar{\theta})\right)\right) \geq c
$$

Now

$$
\bar{u}=\tau_{\bar{m}} \gamma_{0}(\bar{\theta})
$$

$$
\begin{aligned}
& I(\bar{u})<c+\epsilon, \lim _{s \rightarrow \infty} I(\eta(s, \bar{u})) \geq c \text {, and }|\bar{u}(t)|<r_{0} \\
& \quad \text { for all } t<\tilde{t}+t_{m^{*}}+M \text { and all } t>\tilde{t}+t_{m^{\prime}}-M .
\end{aligned}
$$

By Lemma 2.3 and the definition of $\epsilon$,

$$
\left\|I^{\prime}(\eta(s, \bar{u}))\right\|<\frac{r_{0}^{2}}{16 B} \text { for all } s>0 \text { and }\left\|I^{\prime}(\eta(s, \bar{u}))\right\| \rightarrow 0 \text { as } s \rightarrow \infty \text {. }
$$

By Lemma 2.7, $\|\eta(s, \bar{u})\|_{L^{\infty}(\mathbb{R})} \geq r_{0}$ for all $s \geq 0$. We will show that

$$
|\eta(s, \bar{u})(t)|<r_{0} \text { for all } s>0, t<\tilde{t}+t_{m^{*}}-M \text { and } t>\tilde{t}+t_{m^{\prime}}+M .
$$

Thus

$$
\|\eta(s, \bar{u})\|_{L^{\infty}\left(\tilde{t}+t_{m *}-M, \tilde{t}+t_{m^{\prime}}+M\right)} \geq r_{0}
$$

for all $s \geq 0$. Defining $u_{n}=\eta(n, \bar{u})$, we obtain a Palais-Smale sequence $\left(u_{n}\right)$ with $\left\|u_{n}\right\| \leq B$ and $\left\|u_{n}\right\|_{L^{\infty}\left(\tilde{t}+t_{m^{\prime}}-M, \tilde{t}+t_{m^{\prime}}+M\right)} \geq r_{0} . \quad\left(u_{n}\right)$ converges along a subsequence weakly and in $L_{l o c}^{\infty}\left(\mathbb{R}, \mathbb{R}^{N}\right)$ to a critical point $v^{+}$of $I$ with

$$
\left\|v^{+}\right\|_{L^{\infty}(A, \infty)} \geq\left\|v^{+}\right\|_{L^{\infty}\left(\tilde{t}+t_{m^{*}}-M, \tilde{t}+t_{m^{\prime}}+M\right)} \geq r_{0}
$$

proving Proposition 3.1 and Theorem 1.1.

Let us now prove that $|\eta(s, \bar{u})(t)|<r_{0}$ for all $s \geq 0, t<\tilde{t}+t_{m^{*}}-M$. The proof for $t>\tilde{t}+t_{m^{\prime}}+M$ is similar and is omitted. Suppose to the contrary, that $\left|\eta\left(s^{\prime}, \bar{u}\right)(t)\right| \geq r_{0}$ for some $s^{\prime}>0, t<\tilde{t}+t_{m^{*}}-M$. Define

$$
s_{0}=\min \left\{s>0 \mid\|\eta(s, \bar{u})\|_{L^{\infty}\left(-\infty, \tilde{t}+t_{m^{*}}-M\right)}=r_{0}\right\}
$$

$s_{0}$ is well-defined by (3.9). Let $\hat{t} \in\left[\tilde{t}+t_{m^{*}}, \tilde{t}+t_{m^{*}}+M-1\right]$ with

$$
\left\|\eta\left(s_{0}, u\right)\right\|_{W^{1,2}(\hat{t}, \hat{t}+1)}^{2}<B^{2} / M<r_{0}^{2} / 4
$$


using (2.5). Define

$$
\varphi(t)= \begin{cases}1 ; & t \leq \hat{t} \\ \hat{t}+1-t ; & \hat{t} \leq t \leq \hat{t}+1 \\ 0 ; & t \geq \hat{t}+1\end{cases}
$$

Define

$$
u_{0}=\eta\left(s_{0}, \bar{u}\right), \quad w=\varphi u_{0}
$$

$|\varphi| \leq 1$ and $\left|\varphi^{\prime}\right| \leq 1$, so by Lemma $2.4,\|w\| \leq 2\left\|u_{0}\right\| \leq 2 B$. Now, using (2.4),

$$
\begin{aligned}
I^{\prime}\left(u_{0}\right) w= & \int_{\mathbb{R}} u_{0}^{\prime} \cdot w^{\prime}+u_{0} \cdot w-W^{\prime}\left(t, u_{0}\right) w(t) \mathrm{d} t \\
= & \int_{-\infty}^{\tilde{t}+t_{m^{*}}-M}\left|u_{0}^{\prime}\right|^{2}+\left|u_{0}\right|^{2}-W^{\prime}\left(t, u_{0}\right) u_{0}(t) \mathrm{d} t \\
& +\int_{\tilde{t}+t_{m^{*}-M}}^{\hat{t}}\left|u^{\prime}\right|^{2}+\left|u_{0}\right|^{2}-W^{\prime}\left(t, u_{0}\right) u_{0}(t) \mathrm{d} t \\
& +\int_{\hat{t}}^{\hat{t}+1} u_{0}^{\prime} \cdot\left(\varphi u_{0}\right)^{\prime}+\varphi(t)\left|u_{0}\right|^{2}-W\left(t, \varphi u_{0}\right) \varphi u_{0} \mathrm{~d} t \\
\geq & \frac{1}{2} \int_{-\infty}^{\tilde{t}+t_{m^{*}}-M}\left|u_{0}^{\prime}\right|^{2}+\left|u_{0}^{\prime}\right|^{2} \mathrm{~d} t+\frac{1}{2} \int_{\tilde{t}+t_{m^{*}}-M}^{\hat{t}}\left|u_{0}^{\prime}\right|^{2}+\left|u_{0}^{\prime}\right|^{2} \mathrm{~d} t \\
& +\int_{\hat{t}}^{\hat{t}+1} u_{0}^{\prime} \cdot\left(\varphi u_{0}^{\prime}+\varphi^{\prime} u_{0}\right)+\varphi u_{0}^{2}-\frac{1}{2} \varphi^{2} u_{0}^{2} \mathrm{~d} t \\
\geq & \frac{1}{2}\left\|u_{0}\right\|_{L^{\infty}\left(-\infty, \tilde{t}+t_{m} *-M\right)}^{2}-\int_{\hat{t}}^{\hat{t}+1}\left|u_{0}^{\prime}\right|^{2}+u_{0}^{2} \mathrm{~d} t \geq \frac{1}{2} r_{0}^{2}-\frac{1}{4} r_{0}^{2}=\frac{1}{4} r_{0}^{2} .
\end{aligned}
$$

Thus $\left\|I^{\prime}\left(u_{0}\right)\right\| \geq\left|I^{\prime}\left(u_{0}\right) w\right| /\|w\| \geq r_{0}^{2} /(8 B)$, contradicting (3.14). Proposition 3.1, and hence Theorem 1.1, is proven.

\section{REFERENCES}

[1] V. Coti Zelati and P. Rabinowitz, Homoclinic orbits for second order Hamiltonian systems possessing superquadratic potentials. J. Amer. Math. Soc. 4 (1991) 693-627.

[2] P. Montecchiari, M. Nolasco and S. Terracini, Multiplicity of homoclinics for a class of time recurrent second order Hamiltonian systems. Calc. Var. 5 (1997) 523-555.

[3] P. Rabinowitz, Minimax Methods in Critical Point Theory with Applications to Differential Equations, C. B. M. S. Regional Conf. Series in Math. 65. Amer. Math. Soc., Providence (1986).

[4] G. Spradlin, Existence of solutions to a Hamiltonian system without convexity condition on the nonlinearity. Electronic J. Diff. Equ. 2004 (2004) 1-13.

[5] E. Serra, M. Tarallo and S. Terracini, On the existence of homoclinic solutions to almost periodic second order systems. Ann. l'Instit. Henri Poincaré 13 (1996) 783-812. 